\title{
Types for X10 Clocks
}

\author{
Francisco Martins \\ LaSIGE \& University of Lisbon \\ Portugal \\ fmartins@di.fc.ul.pt
}

\author{
Vasco T. Vasconcelos \\ LaSIGE \& University of Lisbon \\ Portugal \\ vv@di.fc.ul.pt
}

\author{
Tiago Cogumbreiro \\ LaSIGE \& University of Lisbon \\ Portugal \\ cogumbreiro@di.fc.ul.pt
}

\begin{abstract}
X10 is a modern language built from the ground up to handle future parallel systems, from multicore machines to cluster configurations. We take a closer look at a pair of synchronisation mechanisms: finish and clocks. The former waits for the termination of parallel computations, the latter allow multiple concurrent activities to wait for each other at certain points in time. In order to better understand these concepts we study a type system for a stripped down version of X10. The main result assures that well typed programs do not run into the errors identified in the X10 language reference, namely the ClockUseException. The study will open, we hope, doors to a more flexible utilisation of clocks in the X10 language.
\end{abstract}

\section{Introduction}

New high-level concurrency primitives are needed more than ever, now that multicore machines lay on our desks and laps. One such primitive is clocks, a generalisation of barriers introduced in the X10 programming language [2]. Barriers are a collective synchronisation mechanism common in Single Program Multiple Data (SPMD) programs [3, 12]. Distinct from synchronisation mechanisms like locks and monitors that let the programmer think about the access to a resource, barriers allow reasoning about process workflow. Clocks are a sophisticated form of barriers that feature dynamic sets of participants for more dynamic programming paradigms, and a two-phase synchronisation (or fuzzy barrier) for improved processor utilisation [5]. The construct is integrated in the X10 language with a promise that it cannot introduce deadlocks. Another primitive, finish, causes an activity to block (i.e., to suspend its execution) until all its sub-activities have completed. Dynamic, unbounded spawning of activities and the finish construct enable fork/join parallelism. The fifth version of language Cilk introduced a specialised "work-stealing" scheduler algorithm that takes advantage of the fork/join model to yield highly efficient language [4]. This style of parallel programming and its run-time system (the work-stealing scheduler) were then incorporated in mainstream languages, e.g., the fork/join framework proposed for Java 7 [6].

Even though the X10 language specification [8] provides a clear, plain English, description of the intended semantics (and properties) of the language, and a formalisation of the semantics [9] allows to prove a deadlock freedom theorem, we decided to investigate a simpler setting in which similar results could be obtained. The aim is not only to obtain a progress property for typable programs based on a simple type system, but also to hopefully provide for clock-safe extensions of the X10 language itself.

Towards this end, we have stripped X10 from most of its features, ending up with a simple concurrent language equipped with finish and the full functionality of X10 clocks, which we call "X10 restricted to clocks," $\left.\mathrm{X} 10\right|_{\text {clocks }}$ for short. For this language we have devised a simple operational semantics with thread (or activity as called in X10) local and global views of (heap allocated) clocks. We have also crafted a simple type system, based on singleton types, drawing expertise from previous work on low-level programming languages [13]. Typable programs are exempt from clock related errors; we conjecture that typable programs enjoy a form of progress property.

K. Honda and A. Mycroft (Eds.): Programming Language Approaches to Concurrency and communication-cEntric Software 2010 (PLACES'10)

EPTCS 69, 2011, pp. 111-129 doi 10.4204/EPTCS.69.8 (c) F. Martins, V. T. Vasconcelos \& T. Cogumbreiro

This work is licensed under the Creative Commons Attribution License. 
Saraswat and Jagadeesan study the X10 programming model, by presenting a formal model of the language that includes clocks, async/finish, conditional atomic blocks, and a hierarchic shared memory [9]. The authors formalise the semantics of the language with a small-step operational semantics, define a bisimulation, and establish that X10 programs without conditional atomic blocks do not deadlock. Lee and Palsberg present a core model for X10, an imperative language augmented with async/finish and atomic blocks suited for inter-procedural analysis through type inference [7]. The authors present an operational semantics based on [9] and a type system that identifies may-happen-parallelism, further explored in [1]. Java features, since version 5, cyclic barriers, java util . concurrent. CyclicBarrier. Unlike X10 clocks these barriers have a fixed set of participants, defined at initialisation time.

This paper constitutes an archival version (post-proceedings) of a workshop paper produced in late 2009. Three of the language extensions proposed at that time have been incorporated in the X10 language definition [8], further discussed in Section 4. They are:

- Aliasing, introduced in version 2.01, January 2010. Prior versions imposed a restriction on how clock values could be aliased, "The initializer for a local variable declaration of type Clock must be a new clock expression. Thus X10 does not permit aliasing of clocks" (page 123, version 2.00).

- Clocks can be transmitted to a spawned activity if they are created in the scope of the enclosing finish, introduced in version 2.05, July 2010. Before we could read, "While executing S [the body of a finish], an activity must not spawn any clocked asyncs' 1 (page 141, version 2.04). Shirako et al. included this extension even prior to our work [10].

- Resume state inheritance, introduced in version 2.05, July 2010. Resumed clocks can be transmitted to forked activities. Prior to version 2.05 we could read, "It is a static error if any activity has a potentially live execution path from a resume statement on a clock c to a async spawn statement (which registers the new activity on c) unless the path goes through a next statement" (page 153, version 2.04).

In summary, the contributions of this work are:

- a simple operational semantics for activities, finish, and clocks that allows to better understand these constructs,

- a type systems allowing to prove safety and progress properties (alternative to the constraint-based system [9]), and

- the promise of a more flexible utilization of the clock constructs.

The rest of this paper presents the syntax in Section 2, the (operational) semantics and the notion of run-time errors in Section 3, the type system and some examples in Section 4, and the main result in Section 5. We conclude, in Section 6, discussing an alternative model for the semantics and pointing directions for future work.

\section{Syntax}

Object-oriented and type-safe, X10 provides for support for concurrency, parallelism, and distribution. Of particular interest to us is the finish synchronisation mechanism that waits for the termination of parallel computations and the clock primitive that allows forcing multiple concurrent activities to wait for each other at certain points in time.

\footnotetext{
${ }^{1}$ In X10 a clocked async corresponds to an activity registered with at least one clock upon creation.
} 


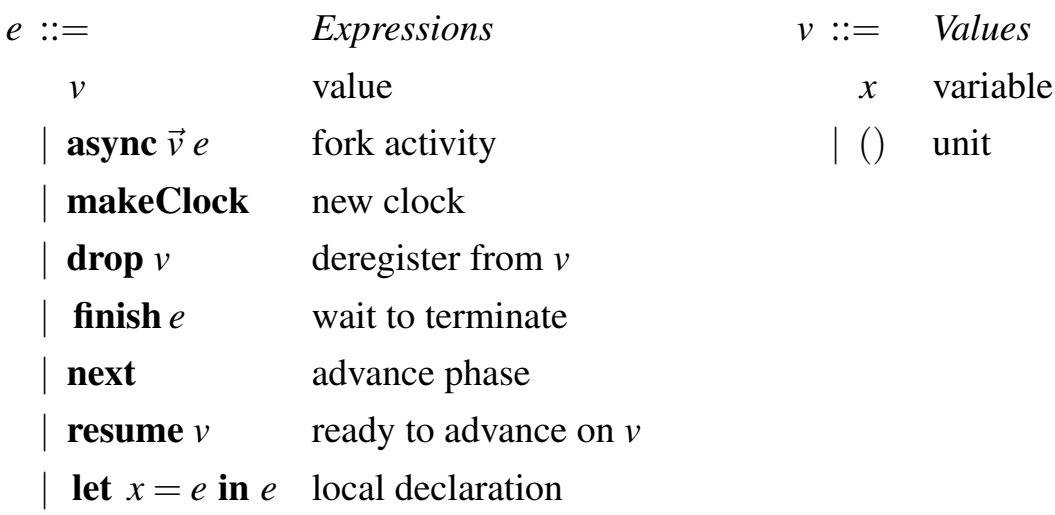

Figure 1: Top-level syntax of $\left.\mathrm{X} 10\right|_{\text {clocks }}$

The top-level, or programmer's, language we address, $\left.\mathrm{X} 10\right|_{\text {clocks }}(\mathrm{X} 10$ restricted to clocks and finish), is a subset of the X10 language, generated by the grammar in Figure 1, and relies on a base set of variables ranged over by $x$. An $\left.\mathrm{X} 10\right|_{\text {clocks }}$ program is an expression $e$ that can operate on activities, clocks, or unit () values. To construct programs we compose expressions through the standard let construct let $x=e_{1}$ in $e_{2}$, which binds variable $x$ to the result of expression $e$ in the scope of expression $e^{\prime}$.

Below we present an example program with the purpose of illustrating the syntax and informally presenting the semantics of the language. The example is composed of three activities an outermost activity $a_{1}$, defined from line 2 to line 16 , an inner activity $a_{2}$, spawned at line 4 , and another inner activity $a_{3}$, spawned at line 5 and lasting until line 11 . Along the example we make use of the derived expression $e_{1} ; e_{2}$ that abbreviates let $x=e_{1}$ in $e_{2}$, where $x$ not free in $e_{2}$.

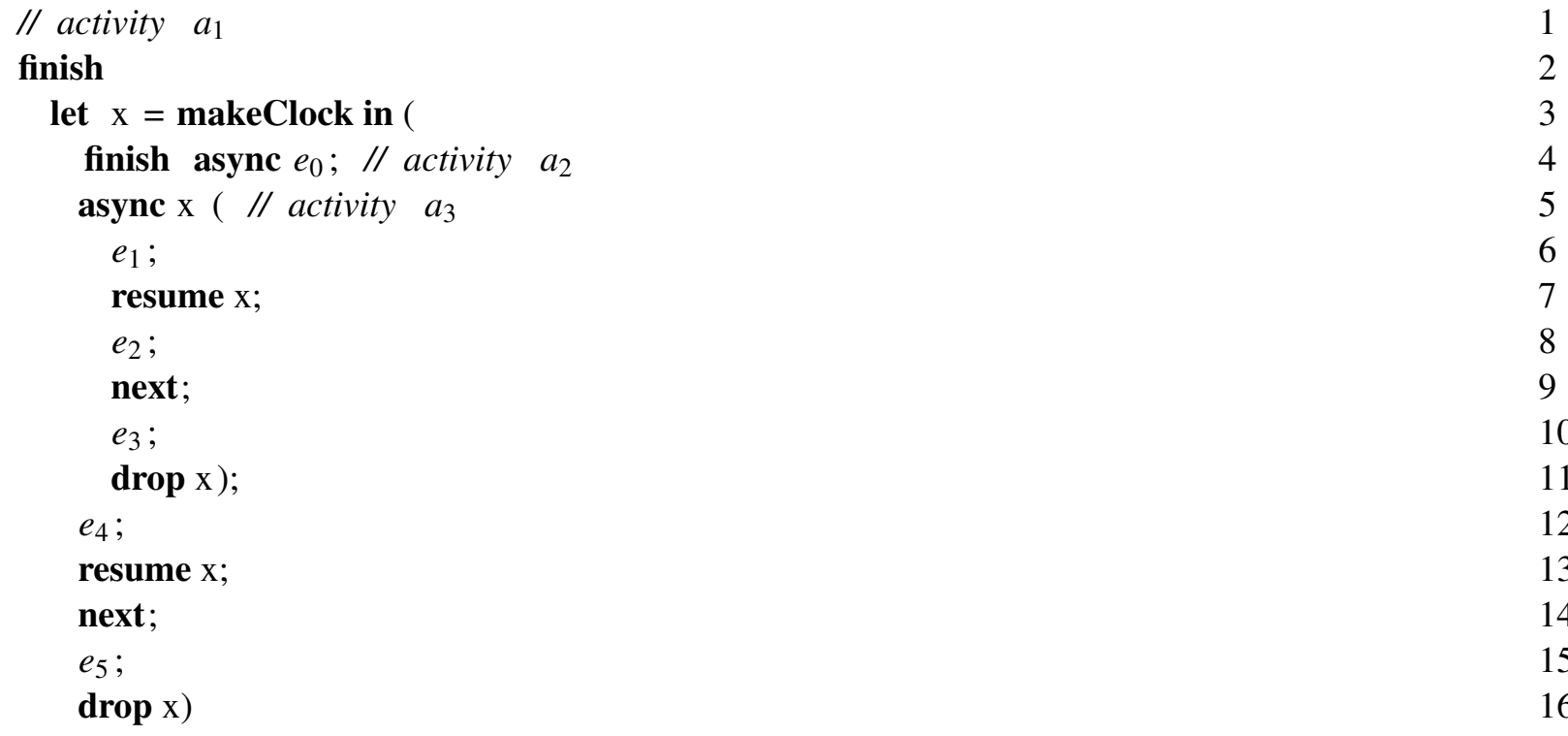

Activities can be registered with zero or more clocks and may share clocks with other activities. A clock can thus count with zero or more different registered activities, which are also called participants. When an activity $a$ is registered with clock $x$, we say that $x$ is a clock held by $a$. Activities may only register themselves with clocks via two different means: when they explicitly create a clock (line 3 , makeClock, creates a clock and registers activity $a_{1}$ with the clock), and when they inherit clocks from 
its parent activity in a spawning operation (line 5 spawns activity $a_{3}$ and registers it with the clock associated with variable $x$ ). Expression drop deregisters an activity from a clock (line 11, drop $x$ deregisters activity $a_{3}$ from clock $x$, whereas line 16 deregisters activity $a_{1}$ from $x$ ). Activities are disallowed to manipulate clocks they are not registered with.

In $\left.\mathrm{X} 10\right|_{\text {clocks, }}$, an activity synchronises via two different methods: by waiting for every activity spawned by expression $e$ to terminate (line 4 only terminates when activity $a_{2}$ and all its sub-activities terminate), and by waiting for its held clocks to advance a phase (activity $a_{3}$ waits at line 9 , whereas activity $a_{1}$ waits at line 14). X10 distinguishes between local and global termination of an expression. Local termination of an expression corresponds to concluding its evaluation (reducing to a value $v$ ). An expression terminates globally when it terminates locally and each activity spawned by the expression has also terminated globally. Expression finish $e$ converts the global termination of expression $e$ into a local termination (line 2 waits for the expression in lines 3-16 to terminate, meaning that it waits as well for the termination of activities $a_{2}$ and $a_{3}$; line 4 waits for activity $a_{2}$ (the result of the evaluation of async $e_{0}$ ) to terminate before launching the sub-activity $a_{3}$, in line 5). The second method to synchronise activities is using clocks. Groups of activities, defined by the participants of a clock, evaluate concurrently until they reach the end of a phase. When every participant of the group reaches the end of the phase, then all move to the next phase, while still executing concurrently. Phases are delimited by expression next; activities evaluate this expression to mark the end of a phase (activities $a_{1}$ and $a_{3}$ synchronise at lines 9 and 14).

An activity can inform all other participants of a clock $x$ that it has completed its phase by using an expression of the form resume $x$, thus making clocks act as fuzzy barriers [5] (line 7). Expression resume can be viewed as an optimisation to diminish contention upon advancing a phase: it allows activities blocked on next to cease waiting for such activities (which can become at most one phase behind the clock's phase). In the example, expression $e_{2}$ might execute at the same time as expression $e_{5}$, since activity $a_{3}$ may trigger (at line 5) activity $a_{1}$ to advance clock $x$ (blocked at line 11), thus evaluating expressions $e_{2}$ and $e_{5}$ in parallel. If we omit expression resume $x$ from this example, then expressions $e_{2}$ and $e_{5}$ cannot evaluate in parallel.

Clocks can be implemented via a (sophisticated) $n$-ary synchronisation mechanism that includes a natural number representing its global phase, initially set to zero. Advancing a clock's phase amounts to incrementing its global phase when every registered activity has quiesced; an activity is quiescent on a clock $x$ after performing a resume $x$. An activity resumes all its held clocks together by evaluating next and suspends itself until these clocks become ready to advance to the next phase.

\section{Operational Semantics}

This section describes the operation semantics of our language, a possible execution of the example described in Section 2, and the notion of run-time errors.

Operational Semantics Figure 2 depicts the run-time syntax of our language. The run-time system relies on one additional set, clock names (or heap addresses, since clocks are the only data structures we allocate in the heap), ranged over by $c$. A state $S$ of an $\left.\mathrm{X} 10\right|_{\text {clocks }}$ computation comprises a shared heap $H$ and a set of named activities $A$ that run concurrently. Activity names, $l$, are taken from the set of variables introduced in Section 2 . The heap stores clock values $h$, triples comprising a natural number $p$ representing its global phase, a set $R$ with the registered activities, and another set $Q$ with the quiesced activities. These sets keep track of the activities that synchronise in the clock $(R)$ and the activities that are 


\begin{tabular}{|c|c|c|c|}
\hline$S::=H ; A$ & States & $e::=$ & Expressions \\
\hline$H::=\left\{c_{1}: h_{1}, \ldots, c_{n}: h_{n}\right\}$ & Heaps & join $l$ & join activity \\
\hline$A::=\left\{l_{1}: a_{1}, \ldots, l_{n}: a_{n}\right\}$ & Sets of named activities & & \\
\hline$h::=\langle p, R, Q\rangle$ & Clock values & $v::=\ldots$ & Values \\
\hline$R, Q::=\left\{l_{1}, \ldots, l_{n}\right\}$ & Sets of activity names & $\mid c$ & clock \\
\hline$a::=(V, e, A)$ & Activities & & \\
\hline$V::=\left\{c_{1}: p_{1}, \ldots, c_{n}: p_{n}\right\}$ & Clocks' local views & $p::=0|1| 2 \mid \ldots$ & Phases \\
\hline
\end{tabular}

Figure 2: Run-time syntax of $\left.\mathrm{X} 10\right|_{\text {clocks }}$

ready to advance the clock to the next phase $(Q)$. Set difference $R \backslash Q$ identifies the activities yet to make progress on a clock; the clock phase only advances when all activities have quiesced (when $R \backslash Q=\emptyset$, equivalently $R=Q$ ). The set of registered activities $R$ also allows to enforce that an activity only operates on registered clocks.

An activity $a$ is composed of a set of clocks' local views $V$, an expression $e$ under execution, and a set of sub-activities $A$. Each activity has its own perception of the global phase of a clock; the clocks' local view $V$ is a map from clock names to natural numbers describing the local phase. The global phase of a clock and that local to one of its activities may diverge in case the activity issues a resume on the clock. Only when the activity issues a next, the local view of the clock and the global phase become in sync. At anytime, a clock's local view is at most one phase behind the global phase. Notice that an activity is itself a tree of activities, since each activity holds a set of (named) sub-activities. When evaluating an expression finish $e$, the activity starts sub-activities for evaluating expression $e$ and all activities spawned by $e$. Otherwise, activities have no sub-activities.

We augment the syntax of expressions at run-time with join $l$. Expression join $l$ results from evaluating finish $e$; label $l$ identifies the activity that executes the body $e$ of the finish expression and that produces the resulting value of the finish $e$ expression.

We present the small step reduction rules for $\left.\mathrm{X} 10\right|_{\text {clocks }}$ in Figures 3 and 4 . Reduction for activities (Figure 3), $H ; a \rightarrow_{l} H^{\prime} ; A ; a^{\prime}$, operates on a heap $H$ and an activity $a$, and produces a possible different heap $H^{\prime}$, a set $A$ of activities spawned during the evaluation of the expression in $a$, and a new activity $a^{\prime}$. Label $l$ is the name of activity $a$.

Rule R-ASYNC is the only rule that affects the set of spawned activities $A$. The programmer specifies a list of clocks $\vec{c}$ on which the new activity is to be registered with. The newly created activity (named $l^{\prime}$ ) is added to the set $R$ of activities registered with clocks $\vec{c}$, and stored in the heap. The result of spawning an activity is the unit value (). The created activity is composed of a clock view holding, for each clock $c$ in $\vec{c}$, a copy of the global phase $p$, an expression $e$ to be evaluated, and an empty set of subactivities. Syntax $H\{c: h\}$ describes a heap $H^{\prime}$ with a distinguished entry $c: h$; formally $H^{\prime}(c)=h$ if $c=c^{\prime}$ else $H(c)$. The new activity inherits each clock $c$ quiescence property, i.e., if $l$ is quiescent on clock $c$ so is $l^{\prime}\left(Q^{\prime}=\right.$ if $l \in Q$ then $Q \cup\left\{l^{\prime}\right\}$ else $Q$ ).

The language specification reads "Clocks are created using a factory method on x10.lang.Clock. The current activity is automatically registered with the newly created clock" [8, page 207]. Expression makeClock creates a new clock in the heap with initial phase 0 , with $l$ as the only registered activity, and with no resumed activities, $\langle 0,\{l\}, \emptyset\rangle$. The activity creating the clock maintains a local clock 


$$
\begin{aligned}
& \{\vec{c}\} \subseteq \operatorname{dom} V \quad l^{\prime} \text { is fresh } \quad Q^{\prime} \triangleq \text { if } l \in Q \text { then } Q \cup\left\{l^{\prime}\right\} \text { else } Q \\
& \overline{H\{c:\langle p, R, Q\rangle\}_{c \text { in } \vec{c}} ;(V, \text { async } \vec{c} e, A) \rightarrow_{l} H\left\{c:\left\langle p, R \cup\left\{l^{\prime}\right\}, Q^{\prime}\right\rangle\right\}_{c \text { in } \vec{c}} ;\left\{l^{\prime}:\left(\{c: p\}_{c \text { in } \vec{c}}, e, \emptyset\right)\right\} ;(V,(), A)} \\
& c \text { is fresh } \\
& \overline{H ;\left(V, \text { makeClock, } A^{\prime}\right) \rightarrow_{l} H\{c:\langle 0,\{l\}, \emptyset\rangle\} ; \emptyset ;\left(V\{c: 0\}, c, A^{\prime}\right)} \\
& Q^{\prime} \triangleq \text { if } p=V(c) \text { then } Q \cup\{l\} \text { else } Q \quad l \notin Q \\
& \overline{H\{c:\langle p, R, Q\rangle\} ;(V, \text { resume } c, A) \rightarrow_{l} H\left\{c:\left\langle p, R, Q^{\prime}\right\rangle\right\} ; \emptyset ;(V,(), A)} \\
& \frac{C_{1} \triangleq\{c \mid V(c)=p, H(c)=\langle p, R, R\rangle\} \quad C_{2} \triangleq\left\{c \mid V(c)=p, H(c)=\left\langle p+1,_{-},\right\rangle\right\} \quad C_{1} \cup C_{2}=\operatorname{dom} V}{H ;(V, \text { next }, A) \rightarrow_{l} H\{c:\langle p+1, R, \emptyset\rangle\}_{c \in C_{1}} ; \emptyset ;\left(\{c: V(c)+1\}_{c \in V},(), A\right)} \\
& \frac{c \in \operatorname{dom} V \quad H^{\prime} \triangleq \text { if } R=\{l\} \text { then } H \backslash\{c\} \text { else } H\{c:\langle p, R \backslash\{l\}, Q \backslash\{l\}\rangle\}}{H\{c:\langle p, R, Q\rangle\} ;(V, \operatorname{drop} c, A) \rightarrow{ }_{l} H^{\prime} ; \emptyset ;(V \backslash\{c\},(), A)} \\
& \frac{l_{0} \text { is fresh }}{H ;(V, \text { finish } e, A) \rightarrow_{l} H ; \emptyset ;\left(V, \text { join } l_{0}, A\left\{l_{0}:(V, e, \emptyset)\right\}\right)} \\
& H ;\left(V \text {, join } l_{0},\left\{l_{0}:\left(\emptyset, v_{0}, \emptyset\right), \ldots, l_{n}:\left(\emptyset, v_{n}, \emptyset\right)\right\}\right) \rightarrow_{l} H ; \emptyset ;\left(\emptyset, v_{0}, \emptyset\right) \\
& \text { (R-ASYNC) } \\
& \text { (R-MAKE) } \\
& H ; A\left\{l:\left(V, \text { let } x=v \text { in } e, A^{\prime}\right)\right\} \rightarrow H ; A\left\{l:\left(V, e[v / x], A^{\prime}\right)\right\} \\
& l \in \operatorname{dom} H \quad H ;(V, e, A) \rightarrow_{l} H^{\prime} ; A^{\prime \prime \prime} ;\left(V^{\prime}, e^{\prime}, A^{\prime}\right) \\
& H ; A^{\prime \prime}\left\{l:\left(V, \text { let } x=e \text { in } e^{\prime \prime}, A\right)\right\} \rightarrow H^{\prime} ; A^{\prime \prime}\left\{l:\left(V^{\prime}, \text { let } x=e^{\prime} \text { in } e^{\prime \prime}, A^{\prime}\right)\right\}, A^{\prime \prime \prime} \\
& \frac{H ; A^{\prime} \rightarrow H^{\prime} ; A^{\prime \prime}}{H ; A\left\{l:\left(V, e, A^{\prime}\right)\right\} \rightarrow H^{\prime} ; A\left\{l:\left(V, e, A^{\prime \prime}\right)\right\}}
\end{aligned}
$$

Figure 4: Reduction rules for states $H ; A \rightarrow H ; A$

view $\{c: 0\}$ stored in $V$. Regarding expression resume the language specification reads "An activity may wish to indicate that it has completed whatever work it wishes to perform in the current phase of a clock c it is registered with, without suspending altogether" (page 208). Rule R-RESUME asserts that when the $l$-labelled activity issues a resume $c$, its label is recorded in the set of resumed activities $R$ if the clock local phase is in sync with the clock global phase $(p=V(c))$; otherwise, the effect of the expression is discarded $(p \neq V(c))$, since the clock has already advance to the next phase. An activity may only perform a resume operation per clock per phase $(l \notin Q)$.

The language specification reads "Execution of this statement [next] blocks until all the clocks that the activity is registered with (if any) have advanced. (The activity implicitly issues a resume on all clocks it is registered with before suspending.) [. . .]. An activity blocked on next resumes execution once it is marked for progress by all the clocks it is registered with" (page 209). In our model, for simplicity's sake, the programmer must issue a resume on all held clocks before expression next. As rule R-NEXT states, expression next blocks the activity until all clocks have been resumed $\left(C_{1}\right)$ or have 
already advance their phases $\left(C_{2}\right)$. Notice that when activities are waiting on a clock $c$, the clock can be in one of three states: (a) there are non-quiescent activities on the clock and $c$ is neither a member of $C_{1}$ nor of $C_{2}$; (b) all registered activities are quiescent on the clock, and so $c$ is a member of $C_{1}$; (c) the clock has advanced to the next phase thus becoming a member of $C_{2}$. When an activity advances a clock global phase, it stops being a member of set $C_{1}$ and becomes a member of set $C_{2}$ for the remaining activities waiting on that clock. Since rule R-NEXT only updates the clock phase of those clocks belonging to $C_{1}$ $\left(H\{c:\langle p+1, R, \emptyset\rangle\}_{c \in C_{1}}\right)$ it ensures that the global clock state is updated only once.

The language specification reads "An activity may drop a clock by executing $c$.drop (). The activity is no longer considered registered with this clock" (page 209). With expression drop $c$, the $l$-labelled activity cedes its control over clock $c$ : we remove $c$ from clock view $V$, and remove activity identifier $l$ from both sets $R$ and $Q$. Two consequences of dropping a clock $c$ are: a) activities waiting on clock $c$ are no longer blocked because of this activity; $b$ ) when executing a next expression, this activity no longer waits for clock $c$. In case $l$ is the only activity registered with clock $c$, it is safe to deallocate the clock, so that the clock's heap space can be reclaimed without resorting to garbage collection. The language specification reads "An activity A executes finish $S$ by executing $S$ and then waiting for all activities spawned by S (directly or indirectly [. . . ]) to terminate" (page 196). Expression finish $e$ creates a child activity and evaluates into expression join $l_{0}$ (rule R-FINISH), which in turn blocks while there exist sub-activities running. When all sub-activities have reduced to a value, activity $l$ (join $l_{0}$ ) evaluates to the value in its sub-activity $l_{0}$ and garbage collects all other sub-activities (rule R-JOIN).

The reduction for states (Figure 4), $S \rightarrow S^{\prime}$, allows for non-deterministic choice of which activity $l$ to evaluate (rule R-ACTIVITY), capturing the concurrency present in X10 computations. We evaluate the let binding from left-to-right (rule R-LET), when the left-hand-side expression becomes a value, we substitute this value for variable $x$ in the continuation expression $e$ (rule R-LET-VAL).

Example Recall the example from Section 2. Consider a loading function that sets up the initial state from a given expression, which in this case is $S_{0}$, an empty heap and an activity evaluating the code in the example under a dummy let. State $S_{0}$ reduces in two steps, using rules R-FINISH and R-LET. The grey boxes highlight a redex and also the corresponding contractum.

$$
S_{0}=\emptyset ;\left\{l_{1}:(\emptyset, \text { let } z=\underbrace{\text { finish let } x=\text { makeClock in }\left(\text { finish }\left(\text { async } e_{0}\right) ; e_{6}\right)}_{\text {Example from Section } 2} \text { in }(), \emptyset)\right\}
$$

where $e_{6}$ is (async $x\left(e_{1} ;\right.$ resume $\left.\left.x ; e_{2} ; \operatorname{next} ; e_{3} ; \operatorname{drop} x\right)\right)$; resume $x ; e_{4} ; \operatorname{next} ; e_{5} ; \operatorname{drop} x$. We perform two reduction steps to illustrate the effect of expression finish on the sub-activities of $l_{1}$.

$$
\left.\emptyset ;\left\{l_{1}:\left(\emptyset \text {, let } z=\text { join } l_{2} \text { in }(),\left\{l_{2}:\left(\emptyset \text {, let } x=\text { makeClock in (finish }\left(\text { async } e_{0}\right) ; e_{6}\right), \emptyset\right)\right\}\right)\right\}
$$

From this state on, while join remains blocked, we apply rule R-ACTIVITY to evaluate the child activities of $l_{1}$. We perform four further reduction steps (R-ACTIVITY, R-LET, R-MAKE, and R-LET-VAL) and observe how expression makeClock updates the heap and the clock view of activity $l_{2}$.

$\left\{c:\left\langle 0,\left\{l_{2}\right\}, \emptyset\right\rangle\right\} ;\left\{l_{1}:\left(\emptyset\right.\right.$, let $z=$ join $l_{2}$ in ()$,\left\{l_{2}:\left(\{c: 0\}\right.\right.$, let $x=c$ in $\left(\right.$ finish $\left(\right.$ async $\left.\left.\left.\left.\left.\left.e_{0}\right) ; e_{6}\right), \emptyset\right)\right\}\right)\right\}$

The non-determinism of our semantics now allows for various different reductions. A possible outcome of a (multi-step) reduction is

$$
\begin{array}{r}
\left\{c:\left\langle 0,\left\{l_{2}, l_{3}\right\}, \emptyset\right\rangle\right\} ;\left\{l_{1}:\left(\emptyset, \text { let } z=\text { join } l_{2} \text { in }(),\left\{l_{2}:\left(\{c: 0\},\left(\text { resume } c ; \operatorname{next} ; e_{5} ; \operatorname{drop} c\right), \emptyset\right),\right.\right.\right. \\
\left.\left.\left.l_{3}:\left(\{c: 0\},\left(\text { resume } c ; e_{2} ; \operatorname{next} ; e_{3} ; \operatorname{drop} c\right), \emptyset\right)\right\}\right)\right\}
\end{array}
$$




$$
\begin{aligned}
& H ; A\left\{l:\left(V \text {, let } x=\operatorname{async} \vec{c} e \text { in } e^{\prime}, A^{\prime}\right)\right\} \in \text { Error if } \vec{c} \nsubseteq \operatorname{dom} H \text { or } c \notin \operatorname{dom} V \text { (E-ASYNC) } \\
& H\left\{c:\left\langle_{-},{ }_{-}, Q\right\rangle\right\} ; A\left\{l:\left(V \text {, let } x=\text { resume } c \text { in } e, A^{\prime}\right)\right\} \in \text { Error if } l \in Q \text { or } c \notin \operatorname{dom} H \text { or } c \notin \operatorname{dom} V \\
& \text { (E-RESUME) } \\
& H ; A\left\{l:\left(V \text {, let } x=\operatorname{drop} c \text { in } e, A^{\prime}\right)\right\} \in \text { Error if } c \notin \operatorname{dom} H \text { or } c \notin \operatorname{dom} V \quad \text { (E-DROP) } \\
& H ; A\left\{l:\left(V \text {, let } x=\text { next in } e, A^{\prime}\right)\right\} \in \text { Error if } V(c)=p, H(c)=\left(p,{ }_{-}, Q\right) \text {, and } \\
& l \notin Q \text {, for some } c \\
& H ; A\left\{l:\left(V \text {, let } x=\text { next in } e, A^{\prime}\right)\right\} \in \text { Error if } c \in \operatorname{dom} V \text { and } c \notin \operatorname{dom} H \text {, for some } c \\
& H ; A\left\{l:\left(V, v_{-}\right)\right\} \in \text { Error } \quad \text { if } V \neq \emptyset \\
& \frac{H ; A^{\prime} \in \text { Error }}{H ; A\left\{l:\left({ }_{-},{ }_{-}, A^{\prime}\right)\right\} \in \text { Error }}
\end{aligned}
$$

Figure 5: The set Error of run-time errors

We now illustrate the case when activities $l_{2}$ and $l_{3}$ are evaluating expressions $e_{2}$ and $e_{5}$ concurrently.

$$
\begin{aligned}
& \text { R-ACTIVITY, R-LET } \rightarrow \text { R-RESUME } \rightarrow{ }_{l_{2}} \text { R-ACTIVITY, R-LET-VAL } \rightarrow \\
& \left\{c:\left\langle 0,\left\{l_{2}, l_{3}\right\},\left\{l_{2}\right\}\right\rangle\right\} ;\left\{l_{1}:\left(\emptyset \text {, let } z=\text { join } l_{2} \text { in }(),\left\{l_{2}:\left(\{c: 0\},\left(\operatorname{next} ; e_{5} ; \operatorname{drop} c\right), \emptyset\right)\right. \text {, }\right.\right. \\
& \left.\left.\left.l_{3}:\left(\{c: 0\},\left(\text { resume } c ; e_{2} ; \operatorname{next} ; e_{3} ; \operatorname{drop} c\right), \emptyset\right)\right\}\right)\right\} \\
& \mathrm{R} \text {-ACTIVITY, R-LET } \rightarrow \text { R-RESUME } \rightarrow_{l_{3}} \\
& \left\{c:\left\langle 0,\left\{l_{2}, l_{3}\right\},\left\{l_{2}, l_{3}\right\}\right\rangle\right\} ;\left\{l_{1}:\left(\emptyset, \text { join } l_{2},\left\{l_{2}:\left(\{c: 0\},\left(\text { next } ; e_{5} ; \operatorname{drop} c\right), \emptyset\right)\right. \text {, }\right.\right. \\
& \left.\left.\left.l_{3}:\left(\{c: 0\},\left(() ; e_{2} ; \mathbf{n e x t} ; e_{3} ; \operatorname{drop} c\right), \emptyset\right)\right\}\right)\right\} \\
& \mathrm{R} \text {-ACTIVITY, R-LET } \rightarrow \text { R-NEXT } \rightarrow_{l_{2}} \\
& \left\{c:\left\langle 1,\left\{l_{2}, l_{3}\right\}, \emptyset\right\rangle\right\} ;\left\{l_{1}:\left(\emptyset \text {, let } z=\text { join } l_{2} \text { in }(),\left\{l_{2}:\left(\{c: 1\},\left(() ; e_{5} ; \operatorname{drop} c\right), \emptyset\right)\right. \text {, }\right.\right. \\
& \left.\left.\left.l_{3}:\left(\{c: 0\},\left(() ; e_{2} ; \mathbf{n e x t} ; e_{3} ; \operatorname{drop} c\right), \emptyset\right)\right\}\right)\right\}
\end{aligned}
$$

After activity $l_{3}$ evaluates resume $c$, activity $l_{2}$, which is blocked evaluating next, progresses, thus allowing expressions $e_{2}$ and $e_{5}$ to execute in parallel. Notice that activity $l_{2}$ remains in phase 0 , while activity $l_{3}$ is in phase 1 .

Run-time errors Run-time errors is the smallest set Error of states generated by the rules in Figure 5 The notion is consistent with all the conditions documented to raise exception ClockUseException, as discussed in the X10 language specification report [8]. The type system we present in Section 4 allow us to reject, at compile time, programs that can potentially throw a ClockUseException.

During an async operation, an activity cannot transmit unregistered clocks through its first argument (rule E-ASYNC). Similarly, activities can only perform resume or drop operations on clocks they are registered with (rules E-RESUME and E-DROP). In particular, it constitutes an error for an activity to drop a clock twice, or to resume a clock more than once (for the same phase) or after dropping it. We achieved a fine grained control over the clocks an activity is registered with. Specifically, it is possible to devise, at compile time, whether an activity resumed or dropped all of its held clocks, as manifest 


$$
\tau::=\text { unit } \mid \operatorname{clock}(\alpha) \quad \text { Types }
$$

Figure 6: Syntax of types

$$
\begin{aligned}
& \mathscr{R}, \alpha \vdash \operatorname{clock}(\alpha) \quad \mathscr{R} \vdash \text { unit } \\
& \text { (T-WF-C, T-WF-U) } \\
& \frac{\mathscr{R} \vdash \tau}{\Gamma, x: \tau ; \mathscr{R} \vdash x: \tau} \quad \Gamma, c: \operatorname{clock}(\alpha) ; \mathscr{R}, \alpha \vdash c: \operatorname{clock}(\alpha) \quad \Gamma ; \mathscr{R} \vdash(): \text { unit } \\
& \text { (T-VAR, T-CLOCK-REF, T-UNIT) } \\
& \frac{\Gamma ; \mathscr{R} \vdash v_{1}: \operatorname{clock}\left(\alpha_{1}\right) \quad \cdots \quad \Gamma ; \mathscr{R} \vdash v_{n}: \operatorname{clock}\left(\alpha_{n}\right) \quad \alpha_{i} \neq \alpha_{j}, \text { if } i \neq j \quad \alpha_{i} \text { not in } \Gamma}{\Gamma ; \mathscr{R} \vdash v_{1} \ldots v_{n}:\left\{\alpha_{1}, \ldots, \alpha_{n}\right\}}
\end{aligned}
$$

(T-CLOCK-SEQ)

Figure 7: Typing rules for values and for well-formed types

from our typing rules later. Therefore, it constitutes an error when an activity evaluates a next expression before resuming all its clocks (rule E-NEXT1). It is also an error when upon evaluating a next there is a held clock that is not in the heap (rule E-NEXT2). Furthermore, an activity cannot evaluate to a value without dropping all of its clocks (rule E-ACT). Rule E-ACT-SET allows error propagation from sub-activities.

Earlier versions of the language specification report (until version 2.05) included two additional error conditions we quote:

- "It is a static error if any activity has a potentially live execution path from a resume statement on a clock c to a async spawn statement (which registers the new activity on c) unless the path goes through a next statement" (page 153, version 2.04). (See example 2, Section 4);

- "While executing S [the body of a finish], an activity must not spawn any clocked asyncs. (Asyncs spawned during the execution of S may spawn clocked asyncs.)" (page 141, version 2.04).

Our type system guarantees soundness in presence of these two conditions.

\section{Type System}

This section presents a type system that uses singleton types to track clock usage throughout a program.

For types we rely on an additional base set of singleton types ranged over by $\alpha$. The syntax of types, depicted in Figure 6, introduces the type unit of unit values, and the type $\operatorname{clock}(\alpha)$ of a particular clock. We assign a different type to each clock in order to ensure the correct usage of the clock constructs within a program.

The type system for $\left.\mathrm{X} 10\right|_{\text {clocks }}$ programs is defined in Figures 7 and 8 , A typing $\Gamma$ is a map from variables (or activity labels) and clocks to types. We write $\operatorname{dom} \Gamma$ for the domain of $\Gamma$. When $x \notin \operatorname{dom} \Gamma$ we write $\Gamma, x: \tau$ for the typing $\Gamma^{\prime}$ such that $\operatorname{dom} \Gamma^{\prime}=\operatorname{dom} \Gamma \cup\{x\}, \Gamma^{\prime}(x)=\tau$, and $\Gamma^{\prime}(y)=\Gamma(y)$ for $y \neq x$. The type system also uses sets of singleton types, ranged over by $\mathscr{R}$, for registered clocks, and $\mathscr{Q}$, for quiescent clocks. 


$$
\begin{aligned}
& \frac{\Gamma ; \mathscr{R} \vdash v: \tau}{\Gamma ; \mathscr{R} ; \mathscr{Q} \vdash v:(\tau, \mathscr{R}, \mathscr{Q})} \quad \frac{\alpha \text { is fresh }}{\Gamma ; \mathscr{R} ; \mathscr{Q} \vdash \text { makeClock: }(\operatorname{clock}(\alpha), \mathscr{R} \cup\{\alpha\}, \mathscr{Q})} \quad \text { (T-VALUE, T-MAKE) } \\
& \Gamma ; \mathscr{R} \vdash v: \operatorname{clock}(\alpha) \quad \alpha \notin \mathscr{Q} \quad \Gamma ; \mathscr{R} \vdash v: \operatorname{clock}(\alpha) \\
& \overline{\Gamma ; \mathscr{R} ; \mathscr{Q} \vdash \text { resume } v:(\text { unit }, \mathscr{R} ; \mathscr{Q} \cup\{\alpha\})} \quad \overline{\Gamma ; \mathscr{R} ; \mathscr{Q} \vdash \text { drop } v:(\text { unit }, \mathscr{R} \backslash\{\alpha\}, \mathscr{Q} \backslash\{\alpha\})} \\
& \frac{\Gamma ; \mathscr{R} \vdash \vec{v}: \mathscr{R}^{\prime} \quad \Gamma ; \mathscr{R}^{\prime} ; \mathscr{Q} \cap \mathscr{R}^{\prime} \vdash e:\left({ }_{-}, \emptyset, \emptyset\right)}{\Gamma ; \mathscr{R} ; \mathscr{Q} \vdash \text { async } \vec{v} e:(\text { unit }, \mathscr{R} ; \mathscr{Q})} \quad \Gamma ; \mathscr{R} ; \mathscr{R} \vdash \text { next: }(\text { unit }, \mathscr{R} ; \emptyset) \quad(\text { T-ASYNC,T-NEXT) } \\
& \frac{\Gamma ; \emptyset ; \emptyset \vdash e:(\tau, \emptyset, \emptyset)}{\Gamma ; \mathscr{R} ; \mathscr{Q} \vdash \text { finish } e:(\tau, \mathscr{R} ; \mathscr{Q})} \\
& \frac{\Gamma ; \mathscr{R} ; \mathscr{Q} \vdash e_{1}:\left(\tau, \mathscr{R}^{\prime}, \mathscr{Q}^{\prime}\right) \quad \Gamma, x: \tau ; \mathscr{R}^{\prime} ; \mathscr{Q}^{\prime} \vdash e_{2}:\left(\tau^{\prime}, \mathscr{R}^{\prime \prime}, \mathscr{Q}^{\prime \prime}\right)}{\Gamma ; \mathscr{R} ; \mathscr{Q} \vdash \text { let } x=e_{1} \text { in } e_{2}:\left(\tau^{\prime}, \mathscr{R}^{\prime \prime}, \mathscr{Q}^{\prime \prime}\right)}
\end{aligned}
$$

Figure 8: Typing rules for expressions

The typing rules for values and for well formed types (Figure 7) are simple to follow. Well-formedness for clock types (rule T-WF-C) ensures that activities only make use of clocks they are registered with. Rule T-CLOCK-SEQ ensures that different clocks (as those in the heap) have distinct singleton clock types, a property that is crucial for establishing type safety. For typing expressions we use a type system (Figure 8) that records the changes made to the set of registered clocks, either by creating or dropping clocks, and to the set of quiescent clocks (using resume and next) of an expression. Typing judgements are of the form $\Gamma ; \mathscr{R} ; \mathscr{Q} \vdash e:\left(\tau, \mathscr{R}^{\prime}, \mathscr{Q}^{\prime}\right)$ meaning that expression $e$ is well typed assuming the types for the free identifiers in $\Gamma$, the registered clocks in $\mathscr{R}$, and the quiescent clocks in $\mathscr{Q}$. The type of an expression is a triple recording the type $\tau$ of its value, as well as the registered $\mathscr{R}^{\prime}$ and the quiescent $\mathscr{Q}^{\prime}$ sets after execution of the expression.

Most typing rules are straightforward. When creating a clock (rule T-MAKE) we associate a new singleton type $\alpha$ with the clock and include it in set of clocks registered by the activity $(\mathscr{R} \cup\{\alpha\})$. Rule T-RESUME, which asserts that "an activity may invoke resume() only on a clock it is registered with, and has not yet dropped" (page 208, vide rule T-VAR), marks clock $\alpha$ as quiescent. Notice that a clock cannot be resumed more than once for the same phase $(\alpha \notin \mathscr{R})$, contrary to the language reference that reads, "Nothing happens if the activity has already invoked a resume on this clock in the current phase" (page 208). A drop $v$ expression removes clock $v$ from both the sets $\mathscr{R}$ and $\mathscr{Q}$, thus the clock cannot be passed to new activities, be the target of a resume expression, or be dropped again.

For expression async $\vec{c} e$, the language reference reads "Starts a new activity, initially registered with clocks $[\vec{v}]$, and running $[e]$. The activity running this code must be registered on those clocks" (page 207, w.r.t. rule T-ASYNC). Rule T-ASYNC asserts that when an activity spawns another activity registered on a sequence of clocks, the quiescent property of the clocks is preserved by propagating the information about the quiescent clock $\alpha(\mathscr{Q} \cap \mathscr{R})$. Moreover, the new activity must have dropped all its clocks upon termination, contrary to the language reference that reads, "All activities are automatically deregistered from all clocks they are registered with on termination (normal or abrupt)" (page 207). An activity cannot share a clock it does not hold, as noted in the language reference, "lacking that registration, cannot register a sub-activity on it [a clock] with async" (page 208). Expression next marks the end of a phase; it checks that all clocks have been resumed and clears the quiescent clocks for the new phase (rules T-NEXT). 
The finish construct may interfere with clocks and cause programs to deadlock. In order to avoid such situations we prevent the body of a finish $e$ expression $(e)$ from accessing any clock already defined, thus eliminating (nested) dependencies between clocks and finish . Rule T-FINISH also forces $e$ to unregister from all clocks it has created, and therefore finish $e$ has no effect on registered and quiescent clocks. This follows the semantics of the current version of X10 that reads, "Inside of $\mathrm{finish}\{\mathrm{S}\}$, all clocked asyncs must be in the scope an unclocked async" (page 210). Refer to the examples below for further discussion on the deadlock problem. When typing a let expression (rule T-LET), its continuation $e_{2}$ is typed taking into consideration the effects produced by expression $e_{1}$. The type of the let is that of $e_{2}$, as usual.

We have deliberately deviated from the standard X10 semantics in three cases: next, drop, and resume. The reasons for such deviation are: (a) to illustrate the power of singleton types in keeping track of clocks, (b) to simplify the (operational and static) semantics, (c) to enforce a programming discipline that may avoid potential bugs, and (d) because the compiler has enough information to suggest, or automatically insert, code fixes (e.g., by enumerating the clocks that need to be dropped before a next; see examples below).

Below we discuss a few $\left.\mathrm{X} 10\right|_{\text {clocks }}$ programs and the semantic guarantees our type system enforces. We decorate the examples with the typing assumptions $(\Gamma, \mathscr{R}, \mathscr{Q})$ holding for each expression.

Example 1: Aliasing Our first example concerns clock aliasing, only introduced in X10 in version 2.01. The example may read a bit trivial but illustrates more sophisticated aliasing situations, derived for example from procedure calls. Clearly a type system with linear control like the one we are going to present allows to relieve such a restriction.

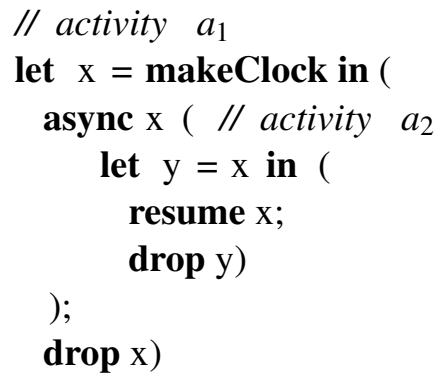

$\begin{array}{lr}/ /\{x: \operatorname{clock}(\alpha)\},\{\alpha\}, \emptyset & 2 \\ / /\{x: \operatorname{lock}(\alpha)\},\{\alpha\}, \emptyset & 3 \\ / /\{x: \operatorname{clock}(\alpha), y: \operatorname{clock}(\alpha)\},\{\alpha\}, \emptyset & 4 \\ / /\{x: \operatorname{clock}(\alpha), y: \operatorname{clock}(\alpha)\},\{\alpha\},\{\alpha\} & 5 \\ / /\{x: \operatorname{clock}(\alpha), y: \operatorname{clock}(\alpha)\}, \emptyset, \emptyset & 6 \\ / /\{x: \operatorname{clock}(\alpha)\},\{\alpha\}, \emptyset & 7 \\ / / \emptyset, \emptyset, \emptyset & 8\end{array}$

In our case the code is typable, assigning the same singleton type $\operatorname{clock}(\alpha)$ to both $x$ and $y$. Upon introducing variable $x$ (line 2), it gets assigned type $\operatorname{clock}(\alpha)$ (vide rules T-LET and T-MAKE). Variable $y$ (line 4 ) gets assigned type $\operatorname{clock}(\alpha)$, the type of $x$ (again using rules T-LET and T-MAKE).

Example 2: Resume state inheritance Our second example deals with a restriction the language reference disallowed up until version 2.04, "A potentially live execution path from a resume statement on a clock c to an async spawn statement" (page 153, version 2.04). Our operational semantics allows the forked activity to inherit the resume state (resumed/not resumed) of the parent activity (vide rule R-ASYNC) and therefore preserves the quiescence property of clocks and avoids a race condition on the clock. Notice that the forked activity $a_{2}$ inherits the quiescent state of clock $\alpha$ (line 4 ), resumed at line 3 .

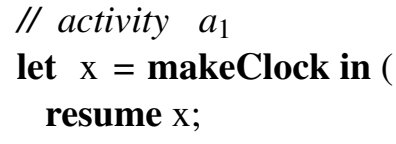

$$
\begin{aligned}
& / /\{x: \operatorname{clock}(\alpha)\},\{\alpha\}, \emptyset \\
& / /\{x: \operatorname{clock}(\alpha)\},\{\alpha\},\{\alpha\}
\end{aligned}
$$



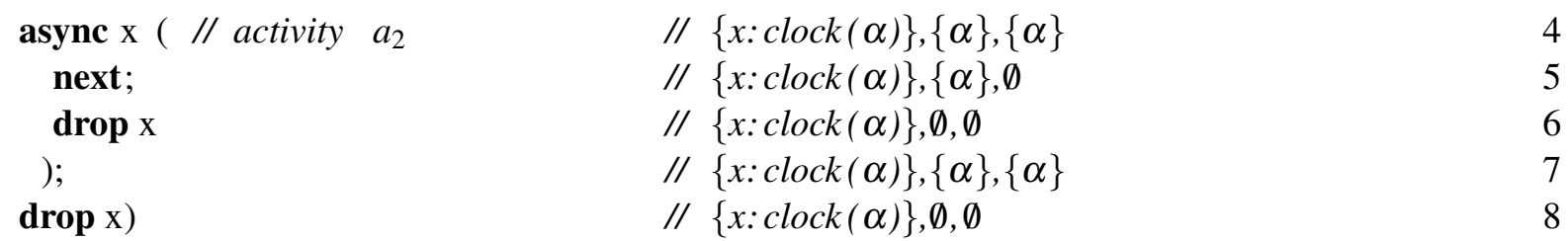

Example 3: Race condition generated by not inheriting the resume state Describes a race condition triggered by clock synchronisation. Activity $a_{1}$ creates a clock $x$, starts a second activity $a_{2}$ registered with clock $x$ that, in turn, resumes on $x$ and starts a third activity $a_{3}$ also registered with $x$.

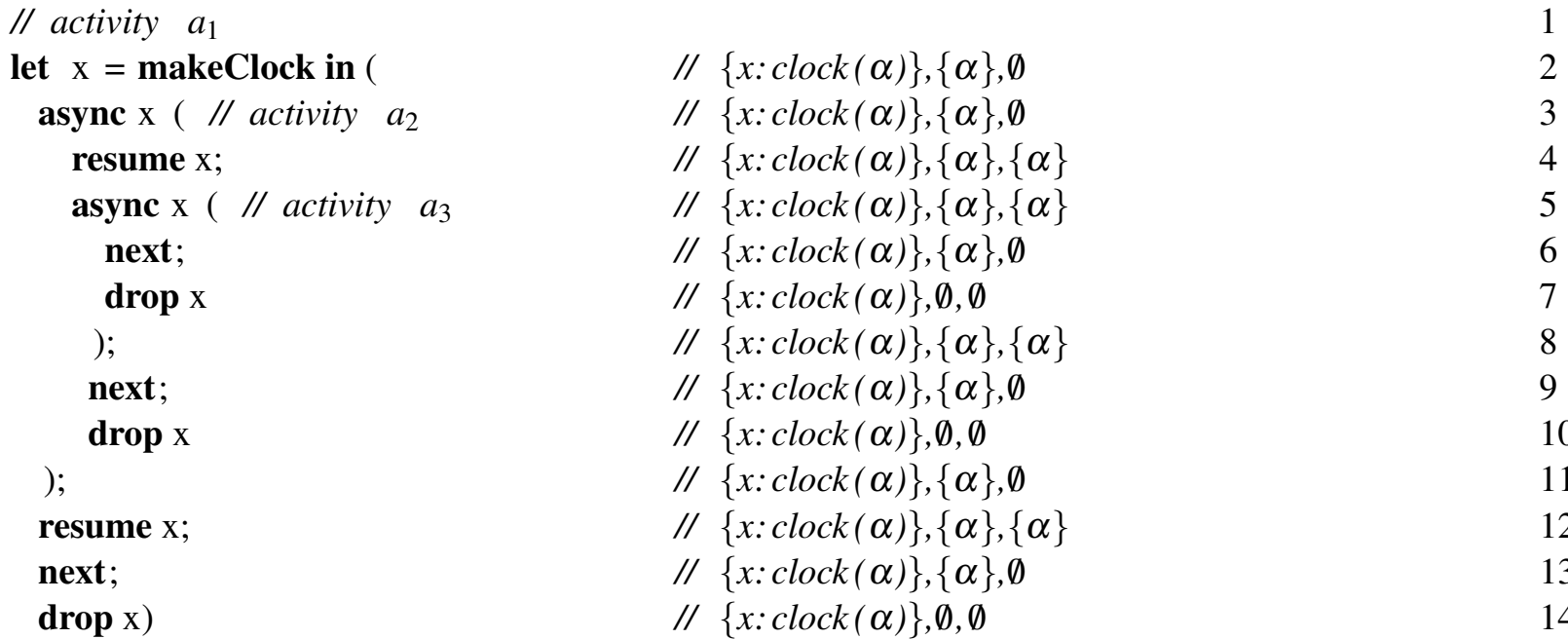

The race condition might occur because after $a_{2}$ resumes on $x$ (line 4), either activity $a_{1}$ may advance clock $x$ phase by executing next (line 13) or $a_{2}$ may register a new activity $a_{3}$ with $x$ (line 5), blocking $a_{1}$ until activity $a_{3}$ executes its next instruction (line 6). By inheriting the resume status of clock $x$, activity $a_{3}$ does not block activity $a_{1}$ and the race condition disappears (vide rule R-ASYNC in Figure 3 and rule T-ASYNC in Figure 8).

Example 4: Resume after resume The next example deals with resuming after resuming, a pattern accepted in X10. Rule T-RESUME rejects the program below, since it is able to determine that clock $x$ is resumed twice.

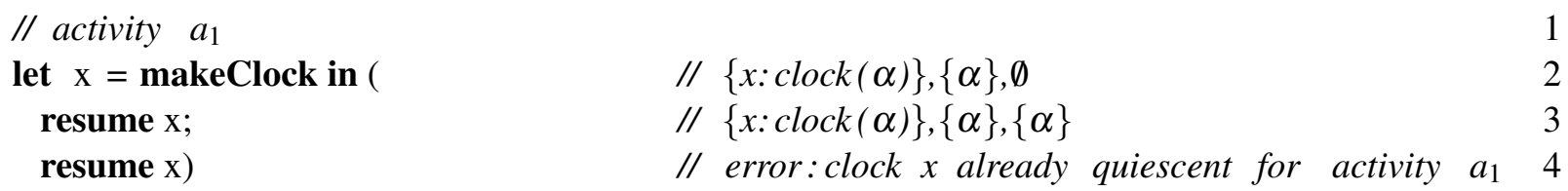

Example 5: Explicit drops Unlike X10, we have decided to explicitly deregister activities from clocks upon activity termination. Our type system keeps track of the clocks an activity is registered with, and rejects programs with activities that finish before deregistering from all its clocks. Clocks without registered activities can be safely garbage collected (vide rule R-DROP). The following example fails to type check, since the launched activity does not drop clock $x$. The compiler may easily suggest an appropriate fix: adding a drop $\mathrm{x}$ after the next instruction on line 5, or even automatically introduce such an instruction. 


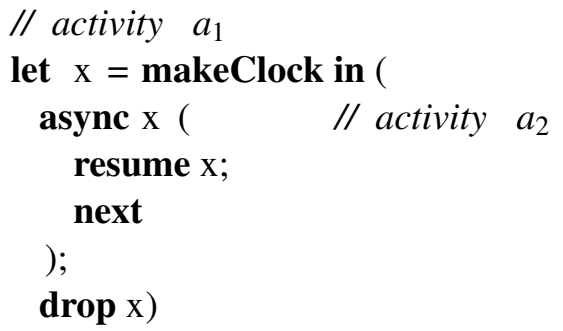

Example 6: Finish/async deadlock Finally, we discuss the interplay among finish, async, and clocks, which may cause programs to deadlock. The following program deadlocks because activity $a_{2}$ is waiting on next (line 6) for activity $a_{1}$ to advance on $x$, which is planned to occur at line 10, but $a_{1}$ is waiting on finish (line 3 ) for activity $a_{2}$ to terminate, so $a_{1}$ never reaches line 10 and the program deadlocks.

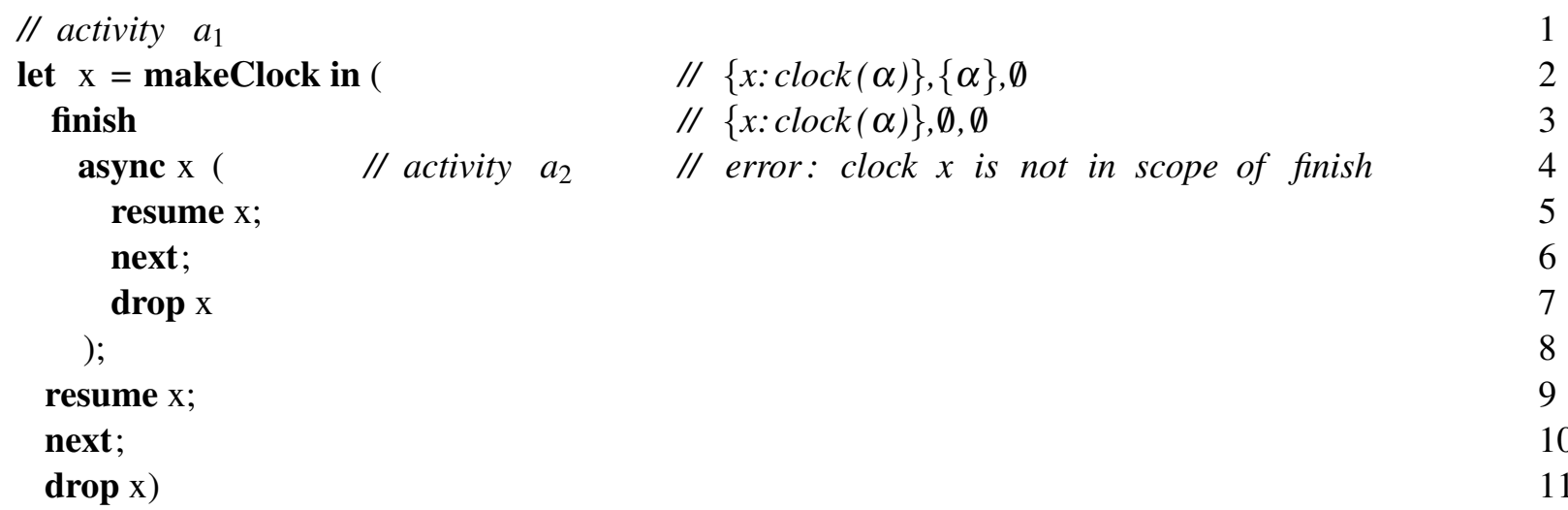

The cause for deadlock is that activity $a_{2}$ is registered with a clock that is defined outside the enclosing finish: clock $x$ is defined in line 2, whereas the finish expression extends from line 3 to line 8 . Our type system rejects this program, because when typing a finish $e$ expression we type check $e$ in an environment with no registered clocks (vide rule R-FINISH).

Example 7: Clocked finish/clocked async Version 2.10 of the X10 language introduces keyword clocked to prefix async and finish.

In the most common case of a single clock coordinating a few behaviors, X10 allows coding with an implicit clock. [...] A clocked finish introduces a new clock. It executes its body in the usual way that a finish does-except that, when its body completes, the activity executing the clocked finish drops the clock, while it waits for asynchronous spawned asyncs to terminate. A clocked async registers its async with the implicit clock of the surrounding clocked finish . [...] Clocked finishes may be nested. The inner clocked finish operates in a single phase of the outer one.

This featured introduced in the language is purely "syntactic sugar," which makes the common practice of creating a single clock and sharing it among activities simpler. The following two code listings present a program with and without the syntactic extension side-by-side. On the left column we show an activity written on a language that resembles X10 [8]. Remember that, in X10, expression next implicitly issues a resume and also that activities implicitly drop all clocks on exit. On the right column we find an equivalent activity written in our language. Activity $a_{1}$ spawns three activities $a_{2}, a_{3}$, and $a_{4}$. Activities $a_{2}$ and $a_{3}$ share the same clock, say $c_{1}$, whereas activity $a_{4}$ holds a different clock, say $c_{2}$, but it 
does not hold $c_{1}$. In the first phase of clock $c_{1}$ the system executes concurrently $e_{1}, e_{3}$, and activity $a_{4}$. In the second phase of clock $c_{1}$, activity $a_{4}$ has terminated, and expressions $e_{2}$ and $e_{4}$ execute concurrently.

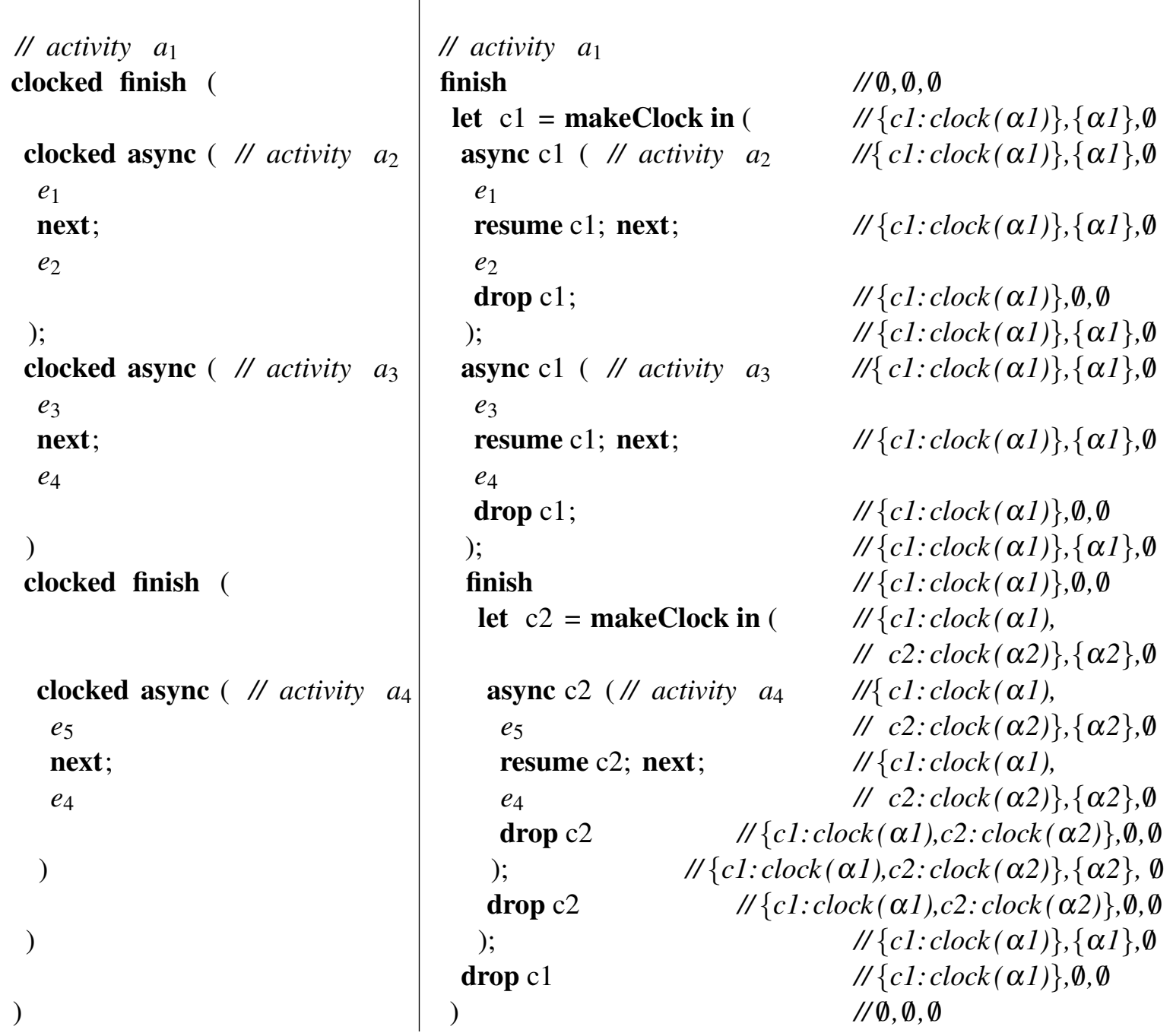

The activity (on the right) obtained by expanding clocked finish and clocked async is still typable with the type system we propose.

\section{Main results}

This section is dedicated to the study of the main result of our system, namely typing preservation and type safety for typable programs.

We are only interested in well-formed states (the rules in Figure 9 check whether a state is wellformed). A state is well formed if for each clock, the set of registered activities with the clock contains exactly those activities that can manipulate it. State

$$
\left\{c:\left\langle_{-}, \emptyset,_{-}\right\rangle\right\} ;\left\{l:\left(\left\{c:{ }_{-}\right\}, \text {let }{ }_{-}=\text {next in }{ }_{-},\right)\right\}
$$

is ill formed, since activity $l$ uses clock $c$ and is not registered with $c$. The activity is able to advance $c$ 's 


$$
\begin{aligned}
& \frac{c \vdash A: S_{1} \quad c \vdash A^{\prime}: S_{2}}{c \vdash A,\left\{l:\left(V,,_{-}, A^{\prime}\right)\right\}: S_{1} \cup S_{2} \cup \operatorname{dom} V \cap\{c\}} \quad c \vdash \emptyset: \emptyset \\
& \text { (WF-ACT-CLOCK, WF-ACT-CLOCK-E) } \\
& \frac{Q \subseteq S \subseteq \operatorname{dom} \Gamma \quad c \vdash A: S \quad \Gamma ; A \vdash H: \diamond}{\Gamma ; A \vdash H,\left\{c:\left\langle{ }_{-}, S, Q\right\rangle\right\}: \diamond} \quad \Gamma ; A \vdash \emptyset: \diamond \quad \text { (WF-HEAP, WF-HEAP-E) } \\
& \frac{H\left(c_{i}\right)=\left\langle{ }_{-}, \mathscr{R}_{i},{ }_{-}\right\rangle \quad l \in \mathscr{R}_{i} \quad H \vdash A: \diamond \quad H \vdash A^{\prime}: \diamond}{H \vdash A,\left\{l:\left(\left\{c_{1}:{ }_{-}, \ldots, c_{n}:{ }_{-}\right\},{ }_{-}, A^{\prime}\right)\right\}: \diamond} \quad H \vdash \emptyset: \diamond \\
& \frac{H \vdash A: \diamond \quad \Gamma ; A \vdash H: \diamond}{\Gamma \vdash H ; A: \diamond}
\end{aligned}
$$

Figure 9: Well-formed states

$$
\Gamma, l: \tau ; \mathscr{R} ; \mathscr{Q} \vdash \text { join } l:(\tau, \mathscr{R}, \mathscr{Q})
$$

Figure 10: Typing rules for run-time expressions

phase without becoming quiescent on $c$. State

$$
\left\{c:\left\langle_{-},\left\{l, l^{\prime}, \ldots\right\},,_{-}\right\rangle\right\} ;\left\{l:\left(\emptyset,_{-},{ }_{-}\right), l^{\prime}:\left(\left\{c:{ }_{-}\right\},,_{-},{ }_{-}\right), \ldots\right\}
$$

is also ill formed, since activity $l$ is mentioned as registered with clock $c$ and is not part of $l$ 's local view (which is $\emptyset$ ). Any other activity registered with $c\left(l^{\prime}\right.$ in the example) is bound to deadlock because $l$ will never quiesce on $c$.

In order to state our results we must be able to type check run-time expressions as well as machine states. The typing rules for run-time expression join $l$ and for machine states and activities are depicted in Figures 10 and 11 . The type of a join $l$ expression (rule T-JOIN) is that of activity $l$. Notice that join $l$ is the result of evaluating a finish $e$ expression (rule R-FINISH) that is, in fact, the type of $e$ (rule TFINISH). It is worth noticing that a heap $H$ is well typed if each clock is assigned to a different singleton type and if the clocks allocated in the heap are exactly those of typing $\Gamma$ (rule T-HEAP where $\mathbf{C}$ is the set of all clocks). Moreover, activities may only resume on registered clocks. An activity $(V, e, A)$ has the type of its expression $e$ (rule T-АCT), which must unregister from all its clocks before terminating, since after evaluating $e$ it is expected that the set of registered clocks should be empty. Rule T-STATE incorporates the definition of well-formed states into the type system. The remaining typing rules should be easy to follow.

Lemma 1 (Weakening). Let a be a variable or a clock name.

1. If $H \vdash A: \diamond$ then $H, c: h \vdash A: \diamond$.

2. If $\Gamma ; \mathscr{R} \vdash v: \tau$ then $\Gamma, a: \tau^{\prime} \vdash v: \tau$.

3. If $\Gamma \vdash V: \mathscr{R}$ then $\Gamma, a: \tau \vdash V: \mathscr{R}$.

4. If $\Gamma ; \mathscr{R} ; \mathscr{Q} \vdash e: T$ then $\Gamma, a: \tau ; \mathscr{R} ; \mathscr{Q} \vdash e: T$. 


$$
\begin{gathered}
\frac{\Gamma \mathscr{R} \vdash c_{1} \ldots c_{n}: \mathscr{R}}{\Gamma \vdash\left\{c_{1}: \ldots, c_{n}:-\right\}: \mathscr{R}} \\
\frac{\Gamma \vdash V: \mathscr{R} \quad \Gamma \in \mathscr{R} \quad \Gamma ; \mathscr{R} ; \mathscr{Q} e:(\tau, \emptyset, \emptyset)}{\Gamma \vdash(V, e, A): \tau} \quad \Gamma \vdash A \\
\frac{\Gamma \vdash a_{1}: \tau_{1} \quad \cdots \quad \Gamma \vdash a_{n}: \tau_{n}}{\Gamma, l_{1}: \tau_{1}, \ldots, l_{n}: \tau_{n} \vdash\left\{l_{1}: a_{1}, \ldots, l_{n}: a_{n}\right\}} \\
\frac{\Gamma \vdash \mathscr{R} \vdash c_{1} \ldots c_{n}: \mathscr{R} \quad\left\{c_{1}, \ldots, c_{n}\right\}=\left.\operatorname{dom} \Gamma\right|_{\mathbf{C}}}{\Gamma \vdash\left\{c_{1}: h_{1}, \ldots, c_{n}: h_{n}\right\}} \\
\frac{\Gamma \vdash H ; A: \diamond \quad \Gamma \vdash H \quad \Gamma \vdash A}{\Gamma \vdash H ; A}
\end{gathered}
$$

Figure 11: Typing rules for machine states

Proof outline. 1. By induction on the derivation of the typing rules. Case WF-ACT-SET-E is direct. For case WF-ACT-SET we use the induction hypothesis to prove that $H,\{c: h\} \vdash A: \diamond$ and $H,\{c: h\} \vdash A^{\prime}: \diamond$; the remaining conditions are given by the hypotheses.

2. By inspecting the typing rules.

3. We apply rule T-VIEW to typify the clocks of the view, then we prove T-CLOCK-SEQ with (2).

4. By induction on the derivation of the typing relation. Cases T-MAKE, T-NEXT, and T-JOIN are direct. Case T-RESUME and T-DROP are proved similarly, using (2) to typify clock $v$. The proof for cases T-FINISH, T-ASYNC, and T-LET follow by induction hypothesis. For case T-ASYNC we also use rule T-CLOCK-SEQ and (2) to typify the clocks of the arguments.

Notice we do not allow heap weakening for it would introduce in the type environment clocks not present in the state.

Lemma 2 (Substitution). If $\Gamma ; \mathscr{R} \vdash v: \tau$ and $\Gamma, x: \tau ; \mathscr{R} ; \mathscr{Q} \vdash e: T$ then $\Gamma ; \mathscr{R} ; \mathscr{Q} \vdash e[v / x]: T$.

Proof outline. For T-VALUE we analyse two cases: when the value is the variable being substituted, and when it is not replaced. For the former case, we apply Lemma 1 on the first hypothesis. For the latter case we use rule T-VAR. Rules T-MAKE, T-NEXT, and T-JOIN are direct. Cases T-DROP and T-RESUME follow by induction hypothesis. Rule T-ASYNC is the most complex. For the clocks being shared we use Lemma 1 and the second hypothesis. For the expression being spawned we apply the induction hypothesis. Rule T-FINISH is proved similarly to T-ASYNC, but simpler, since T-FINISH has no arguments and its set of clocks is empty.

Lemma 3 (Preservation for activities). If $\Gamma \vdash H$ and $\Gamma \vdash V: \mathscr{Q}$ and $\mathscr{Q} \subseteq \mathscr{R}$ and $\Gamma ; \mathscr{R} ; \mathscr{Q} \vdash e: T$ and $\Gamma \vdash A$ and $l \in \operatorname{dom} H$ and $H ;(V, e, A) \rightarrow_{l} H^{\prime} ; A^{\prime \prime} ;\left(V^{\prime}, e^{\prime}, A^{\prime}\right)$, then $\Gamma^{\prime} \vdash H^{\prime}$ and $\Gamma^{\prime} \vdash V^{\prime}: \mathscr{Q}^{\prime}$ and $\mathscr{Q}^{\prime} \subseteq \mathscr{R}^{\prime}$ and $\Gamma^{\prime} ; \mathscr{R}^{\prime} ; \mathscr{Q}^{\prime} \vdash e^{\prime}: T$ and $\Gamma^{\prime} \vdash A^{\prime}, A^{\prime \prime}$, for some $\Gamma^{\prime} \supseteq \Gamma$.

Proof outline. Despite the scary look of the statement, its proof is a routine inspection of the rules in the relation $H ;(V, e, A) \rightarrow_{l} H^{\prime} ; A^{\prime \prime} ;\left(V^{\prime}, e^{\prime}, A^{\prime}\right)$.

Lemma 4 (Preservation for $\left.\mathrm{X} 10\right|_{\text {clocks }}$ ). If $\Gamma \vdash S$ and $S \rightarrow S^{\prime}$ then $\Gamma^{\prime} \vdash S^{\prime}$ and $\Gamma \subseteq \Gamma^{\prime}$. 
Proof outline. By induction on the derivation of the relation $S \rightarrow S^{\prime}$. In all cases we build the derivation tree for $\Gamma \vdash S$ using rules T-STATE, T-ACT-SET, and T-ACT, collect the hypotheses, use the above Lemmas, and then build a tree for $\Gamma^{\prime} \vdash S^{\prime}$ using the same typing rules. The base cases are when the derivation ends with rules R-LET-VAL and R-LET. For R-LET-VAL we take $\Gamma^{\prime}=\Gamma$ and use the substitution Lemma2. For R-LET we use the (specially crafted) preservation for activities (Lemma 3), as well as the weakening (Lemma 1) for the extant activity set $A$. The induction step is when derivation ends with rule R-ACTIVITY; in this case we use the Weakening Lemma.

Theorem 5 (Type Safety). If $\Gamma \vdash S$ and $S \rightarrow^{*} S^{\prime}$, then $S^{\prime} \notin$ Error.

Proof outline. We first establish that $\Gamma^{\prime} \vdash S^{\prime}$ using preservation (Lemma 4). Then we proceed by contradiction. The contradiction is proved by induction on the definition of Error predicate. For the base cases of resume, drop, and async we build the derivation trees for the errors in Figure 5, to conclude that $\Gamma^{\prime} \vdash V: \mathscr{R}$ and $\Gamma^{\prime} ; \mathscr{R} \vdash c$ : $\operatorname{clock}(\alpha)$. Sequent $\Gamma^{\prime} \vdash V: \mathscr{R}$ is derived from rule T-VIEW, which effectively establishes a one-to-one correspondence between the clock names $c$ in $V$ and the singleton types $\alpha$ in $\mathscr{R}$. On the other hand, sequent $\Gamma^{\prime} ; \mathscr{R} \vdash c: \operatorname{clock}(\alpha)$ is derived via rule T-WF-C, which says that $\alpha \in \mathscr{R}$. Since $\alpha \in \mathscr{R}$, the correspondence allows us to conclude that $c \in \operatorname{dom} V$. Establishing that $v \in \operatorname{dom} H$ is easier. Given that $\Gamma^{\prime} \vdash H$, we conclude that $\operatorname{dom} H=\operatorname{dom}\left(\Gamma^{\prime} \mid \mathbf{C}\right)$, and from sequent $\Gamma^{\prime} ; \mathscr{R} \vdash c: \operatorname{clock}(\alpha)$ we know that $c \in \operatorname{dom} \Gamma^{\prime}$, hence done.

In Section 3 we introduced a loading function that builds the initial machine state corresponding to a given expression. Such a state is typable if the expression is. Let load $(e)$ be defined as the state $\emptyset ;\{l:(\emptyset$, let $x=e$ in unit, $\emptyset)\}$. Then we have:

Lemma 6. If $\Gamma ; \emptyset ; \emptyset \vdash e: T$ then $\Gamma \vdash \operatorname{load}(e)$.

Our final result guarantees that a well-typed expression does not reduce to an error.

Corollary 7. If $\Gamma ; \emptyset ; \emptyset \vdash e: T$ then $\operatorname{load}(e)$ does not reduce to an Error.

Proof. From the lemma above and Theorem 5 .

We anticipate a progress property for typable processes. Typability ensures that processes do not get stuck when dropping a clock that is not in its clock set anymore, or when otherwise trying to access a clock that it not allocated in the heap. The remaining case is next where the activity waits for set $C_{1}$ (the set of quiescent clocks the activity is registered with) to grow until becoming (together with $C_{2}$-the set of clocks that have already advance their phase) the clock set of the activity. And this is bound to happen for both next and drop, since in each activity both implicitly resume all clocks. We foresee as well that typability also rules out programs that deadlock, since finish expressions can only use clocks created in its body expressions.

\section{Discussion and future work}

We study two synchronisation constructs of X10: a primitive finish that waits for the termination of activities (lightweight threads), and clocks (a generalisation of barriers). To better understand the language we define an operational semantics and a type system (alternative to the constraint-based system [9]) for a subset of X10 called X10 $\left.\right|_{\text {clocks. }}$. Our main result is type safety for typable programs (Theorem 5).

Our semantics represents clocks in the heap as triples $\langle p, R, Q\rangle$ relying on two sets for recording the registered activities $R$ and the quiesced activities $Q$ on a clock. Implementing operations that work 
with sets is costly; for instance rule R-NEXT needs to compute sets $C_{1}$ and $C_{2}$, by checking if sets $R$ and $Q$ are equal, and then verify if $C_{1} \cup C_{2}=\operatorname{dom} V$. Should we make a real life implementation of the proposed semantics, set operations would have a significant impact on performance. We sketch a much faster approach that chooses to represent clocks as triples $\langle p, r, q\rangle$ describing the clock phase, as before, but taking $r$ and $q$ as the cardinal numbers of sets $R$ and $Q$. With this representation we lose information about the identity of the activities registered with a clock and, in particular, we cannot determine if an activity has already resumed in the current phase (vide rules R-ASYNC and R-RESUME). To overcome this problem we need to enrich the clock local view with an indicator of whether an activity has performed a resume in the current phase. Thus, a clock local view becomes a pair $\langle p, b\rangle$ containing the current clock phase $p$ (as before) and the resume boolean indicator $b$, describing when the activity has resumed. With this information it is straightforward to adapt rules R-ASYNC, R-MAKE, R-RESUME, R-NEXT, and RDROP. For instance, rule R-RESUME only updates the clock global view $(q \leftarrow q+1)$ whenever its local view indicator is false. Also, rule R-NEXT needs to set $r$ to zero when advancing the clock global phase, and to clear the indicator $b$ upon advancing the clock local phase. Checking that all activities registered with a clock have quiesced amounts to compare two integer values $(r=s)$, instead of two sets $R$ and $Q$ as before. The main reasons for not adopting the semantics just sketched are that the chosen semantics needs fewer rules and is easier to read and understand.

We intend to investigate imperative features of the language, specially those related with clocks, and also other language constructs. The finish construct is not only used to wait for the termination of sub-activities, but also, as the language reference reads, "A collection point for uncaught exceptions generated during the execution of $S$ [the body of a finish]" [8, page 196]. Enriching our model with exceptions seems like a natural, promising follow-up of our work. The language report also reads, "X10 does not contain a register statement that would allow an activity to discover a clock in a data structure and register itself on it" (page 208); we would like to study type-safe extensions to the language that might alleviate this restriction in controlled situations. Furthermore, we expect to extend our results to $\left.\mathrm{X} 10\right|_{\text {clocks }}$ equipped with recursion or some form of iteration. Futures are a form of a function that evaluates asynchronously, like an activity, but can be forced to finish locally to return a value. The semantics of a future, in what regards termination, is like the finish construct, but its use cases are different. We would also like to allow futures to register themselves with clocks, a feature missing in $\mathrm{X} 10$.

Phasers are a coordination construct that unifies collective and point-to-point synchronisations with performance results competitive to existing barrier implementations [10]. Phasers can be seen as an extension over clocks that allow for more fine-grained control over synchronisation modes. Phaser accumulators are reduction constructs for dynamic parallelism that integrate with phasers [11]. Although further investigation is needed, we believe our work can be extended to accommodate phasers and phaser accumulators, specially with regards to the operational similarities between clocks and phasers.

\section{Acknowledgements}

The authors would like thank the anonymous referees for constructive criticisms and detailed comments.

\section{References}

[1] Shivali Agarwal, Rajkishore Barik, Vivek Sarkar \& Rudrapatna K. Shyamasundar (2007): Mayhappen-in-parallel analysis of X10 programs. In: Proceedings of PPoPP'07, ACM, pp. 183-193, 
doi $10.1145 / 1229428.1229471$

[2] Philippe Charles, Christian Grothoff, Vijay Saraswat, Christopher Donawa, Allan Kielstra, Kemal Ebcioglu, Christoph von Praun \& Vivek Sarkar (2005): X10: an object-oriented approach to non-uniform cluster computing. In: Proceedings of OOPSLA'05, ACM, pp. 519-538, doi:10.1145/1094811.1094852

[3] Frederica Darema, David A. George, V. Alan Norton \& Gregory F. Pfister (1988): A SingleProgram-Multiple-Data computational model for EPEX/FORTRAN. Parallel Computing 7(1), pp. 11-24, doi:10.1016/0167-8191(88)90094-4.

[4] Matteo Frigo, Charles E. Leiserson \& Keith H. Randall (1998): The implementation of the Cilk-5 multithreaded language. In: Proceedings of PLDI'98, ACM, pp. 212-223, doi $10.1145 / 277650.277725$

[5] Rajiv Gupta (1989): The fuzzy barrier: a mechanism for high speed synchronization of processors. SIGARCH Computer Architecture News 17(2), pp. 54-63, doi:10.1145/68182.68187.

[6] Doug Lea (2000): A Java fork/join framework. In: Proceedings of JAVA'O0, ACM, pp. 36-43, doi: $10.1145 / 337449.337465$.

[7] Jonathan K. Lee \& Jens Palsberg (2010): Featherweight X10: a core calculus for async-finish parallelism. In: Proceedings of PPoPP'10, ACM, pp. 25-36, doi:10.1145/1693453.1693459

[8] Vijay Saraswat (2011): Report on the Programming Language X10, version 2.12. Technical Report, IBM Research.

[9] Vijay Saraswat \& Radha Jagadeesan (2005): Concurrent clustered programming. In: Proceedings of CONCUR'05, LNCS 3653, Springer, pp. 353-367, doi:10.1007/11539452_28

[10] Jun Shirako, David M. Peixotto, Vivek Sarkar \& William N. Scherer (2008): Phasers: a unified deadlock-free construct for collective and point-to-point synchronization. In: Proceedings of ICS'08, ACM, pp. 277-288, doi $10.1145 / 1375527.1375568$

[11] Jun Shirako, David M. Peixotto, Vivek Sarkar \& William N. Scherer (2009): Phaser accumulators: A new reduction construct for dynamic parallelism. In: Proceedings of IPDPS'09, IEEE Computer Society, pp. 1-12, doi:10.1109/IPDPS.2009.5161071.

[12] Chau-Wen Tseng (1995): Compiler optimizations for eliminating barrier synchronization. In: Proceedings of PPOPP'95, ACM, pp. 144-155, doi:10.1145/209936.209952

[13] Vasco T. Vasconcelos, Francisco Martins \& Tiago Cogumbreiro (2010): Type Inference for Deadlock Detection in a Multithreaded Typed Assembly Language. In: Post-proceedings of PLACES'09, EPTCS 17, pp. 95-109, doi 10.4204/EPTCS.17.8 